\title{
Utilization of waste tyres pyrolysis oil vapour in the synthesis of Zeolite Templated Carbons (ZTCs) for hydrogen storage application
}

\author{
NICHOLAS M. MUSYOKA ${ }^{1 *}$, KHAVHARENDWE. M. RAMBAU ${ }^{1,2}$, NCHOLU \\ MANYALA $^{2 *}$, JIANWEI REN $^{1}$, HENRIETTA W. LANGMI $^{1}$, MKHULU K. MATHE $^{1}$ \\ ${ }^{I}$ HySA Infrastructure Centre of Competence, Materials Science and Manufacturing, Council for Scientific and \\ Industrial Research (CSIR), Pretoria, South Africa. \\ ${ }^{2}$ Department of Physics, Institute of Applied Materials, SARCHI Chair in Carbon Technology and Materials, \\ University of Pretoria, South Africa.
}

\begin{abstract}
In this study, we investigated the potential for use of waste tyre pyrolysis oil vapour as a carbon precursor in the synthesis of zeolite templated carbons (ZTC). With Zeolite 13X as the template, the ZTCs were synthesised using two methods namely: 1-step process which involved the carbonization of gaseous carbon precursor in the zeolite template (in this case, ethylene and pyrolysis oil vapour) and the 2-step synthesis method involved the impregnation of zeolite pores with furfural alcohol prior to carbonization of the gaseous carbon precursor. The replication of the zeolite $13 \mathrm{X}$ structural ordering was successful using both methods. The 2-step synthesized ZTCs were found to possess the highest specific surface area $\left(3341 \mathrm{~m}^{2} / \mathrm{g}\right)$ and also had the highest $\mathrm{H}_{2}$ storage capacity (2.5 wt. \%). The study therefore confirmed an additional novel strategy for value-addition of waste tyre pyrolysis by-products.
\end{abstract}

Keywords:. Zeolites; zeolite templated carbon (ZTC); waste tyres; pyrolysis oil vapor

\footnotetext{
*Address correspondence to Nicholas M. Musyoka (Energy Centre, Council for Scientific and Industrial Research (CSIR), Pretoria, South Africa) E-mail:- nmusyoka@csir.co.za or Ncholu Manyala (Department of Physics, Institute of Applied Materials, SARCHI Chair in Carbon Technology and Materials, University of Pretoria, South Africa) E-mail:- ncholu.manyala@up.ac.za.
} 


\section{Introduction}

The increase in waste tyres has led scientists in the quest for finding various innovative ways for minimising problems associated with their disposal. ${ }^{[1]}$ The sought solutions are not only meant to be environmentally friendly but should also be economical. ${ }^{[2]}$ The most efficient way of waste tyre recycling is the process of pyrolysis. This is a thermodynamic process of decomposing waste tyres at high temperatures in the absence of oxygen. The by-products of the pyrolysis process are; pyrolysis gas (pyro-gas), pyrolysis oil and solid char. ${ }^{[3]}$ Even though many studies on beneficiation of pyrolysis by-products have been conducted, ${ }^{[2-4]}$ there is still a need for adding more value to the pyrolysis by-products. The objective of the present study was to investigate the potential for utilising the rich carbonaceous gases obtained from the waste tyre pyrolysis oil to synthesize zeolite templated carbons (ZTCs). To the best of our knowledge, there are no reports in the open literature on the utilization of pyrolysis gas in the production of ZTCs. Zeolite templating process is one of the most preferred ways of producing highly porous carbons with applications in hydrogen storage and batteries. ${ }^{[5,6]}$ The process involves the use of rigid or solid porous materials as a template and a foreign carbonaceous material is introduced into the cavity of the template. The composite is then carbonized and thereafter the template is removed to result to a highly porous carbon material. ${ }^{[7-9]}$ Various carbonaceous sources can be used for the templating process of ZTCs, and in most cases, gaseous carbon precursors such as ethylene, propylene and acetronitrile are commonly used. ${ }^{[8,10-13]}$ However, these gaseous feedstocks are often commercially sourced with high costs. It is on this basis that we investigated the potential for the use of pyrolysis oil vapour as a precursor for the synthesis of ZTCs. The obtained ZTCs were further tested for hydrogen storage applications. 


\section{Materials and methods}

\section{Chemicals and materials}

Commercial zeolite 13X was obtained from Sigma-Aldrich and the pyrolysis oil was supplied by International Rubber Recycling Company based in Pretoria, South Africa. $98 \%$ furfural alcohol (FA) was obtained from Sigma-Aldrich. Ethanol (99.5\%) was supplied by Merck. Hydrochloric acid $(37 \%, \mathrm{HCl})$ and $40 \%$ hydrofluoric acid (HF) were purchased from Associated Chemical Enterprise.

\section{Synthesis of zeolite templated carbons}

The ZTCs were synthesised using two different methods; 1-step method involved the carbonization by gaseous carbon precursor (ethylene or pyrolysis oil vapour). The 2-step method involved the impregnation of zeolite pores with furfural alcohol and thereafter followed by gaseous carbon precursor (ethylene or pyrolysis oil vapour). In both instances, the as-received commercial zeolite was dried under vacuum at $120{ }^{\circ} \mathrm{C}$ for $6 \mathrm{~h}$ in order to remove any trapped or adsorbed water molecules from the zeolite cavities. To generate samples from the 1-step method, zeolite samples were transferred into ceramic boats and placed in a tube furnace and argon/ethylene (or argon/pyrolysis oil vapour) mixture was allowed through the zeolite sample and the chemical vapour deposition (CVD) process was allowed to occur at $700{ }^{\circ} \mathrm{C}$ for $1 \mathrm{~h}$. Carbonization of the deposited carbon took place at 900 ${ }^{\circ} \mathrm{C}$ for $3 \mathrm{~h}$. On the 2-step method, the dried samples were initially impregnated with furfural alcohol. The mixture was then filtered and washed with a small quantity of ethanol using a pipette. The zeolite/FA composite was transferred into the tube furnace and polymerization of the impregnated FA was conducted under argon flow $(500 \mathrm{~mL} / \mathrm{min})$ at $80{ }^{\circ} \mathrm{C}$ for $24 \mathrm{~h}$ and thereafter stabilized for an additional $8 \mathrm{~h}$ at $150{ }^{\circ} \mathrm{C}$. The temperature was thereafter increased to $700{ }^{\circ} \mathrm{C}$ at a heating rate of $5{ }^{\circ} \mathrm{C} / \mathrm{min}$. At this temperature, the ethylene or pyrolysis oil 
vapour was allowed to flow through for $3 \mathrm{~h}$. The carbon-loaded zeolite composite was further carbonized at $900{ }^{\circ} \mathrm{C}$ for an additional $3 \mathrm{~h}$ under the flow of argon gas. In both methods, the pyrolysis oil vapour was produced by opening a by-pass valve that allowed bubbling of argon gas through pyrolysis oil. The experimental set-up for the chemical vapour deposition (CVD) process utilizing of ethylene gas and pyrolysis oil vapor is presented in Figure 1.

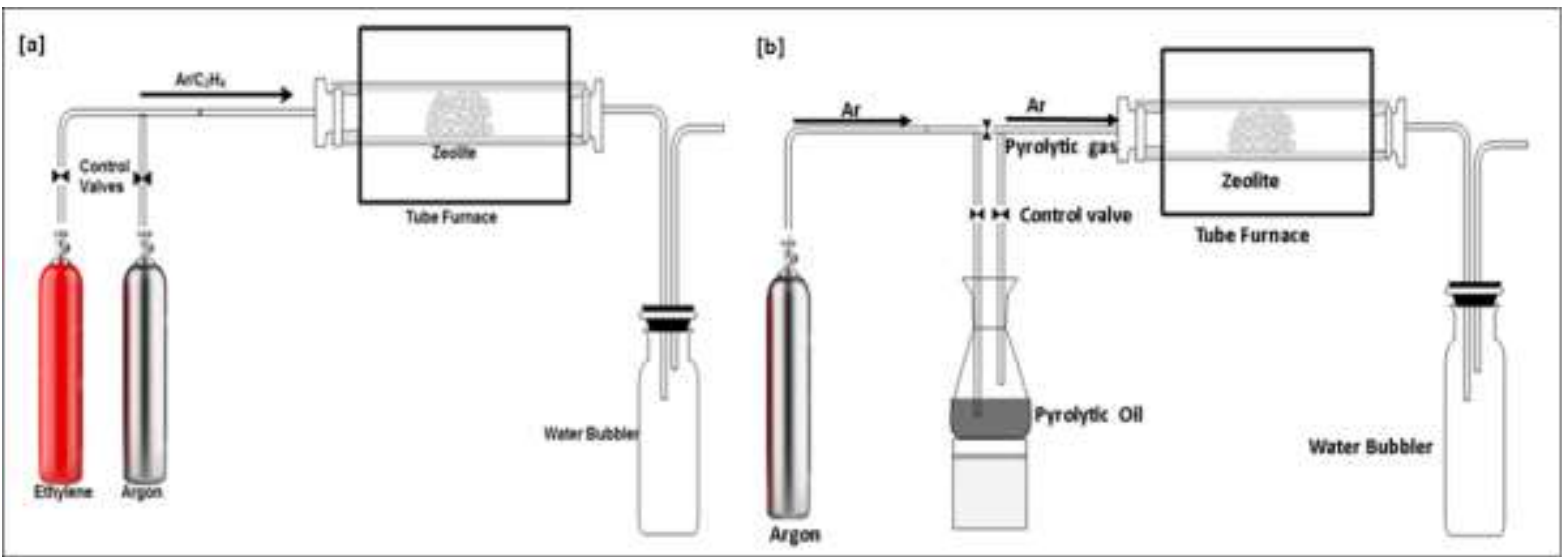

Figure 1: CVD set-up for synthesis of ZTCs using a) ethylene and b) pyrolysis oil vapour as carbon precursors.

Removal of the inorganic framework of the zeolite was facilitated by mixing the resulting zeolite/carbon composites with $10 \%$ hydrofluoric acid (HF) for $3 \mathrm{~h}$ at room temperature under stirring at $250 \mathrm{rpm}$ and further refluxing with $10 \%$ hydrochloric acid $(\mathrm{HCl})$ at $70{ }^{\circ} \mathrm{C}$ for $48 \mathrm{~h}$ under stirring at $250 \mathrm{rpm}$. Resulting samples were filtered and washed thoroughly with copious amount of demineralized water then dried at $90{ }^{\circ} \mathrm{C}$ for $12 \mathrm{~h}$. The synthesised materials were denoted as follows: The 2-step synthesized ZTC sample from FA and ethylene gas was denoted as COMFA-ZTCethy whereas that from pyrolysis oil vapour was symbolized as COMFA-ZTCpy. On the other hand, the 1-step synthesized ZTC from ethylene was denoted as COM-ZTCethy whereas that obtained from pyrolysis oil vapour was symbolized as COM-ZTCpy. 


\section{Analytical methods}

Pyrolysis oil vapour that was collected by bubbling argon through pyrolysis oil was sampled using Tedlar® gas bags and was analysed on the thermal desorption GC-MS using the BTEX method. X-ray diffraction (XRD) patterns were recorded using a PANalytical X'Pert Pro powder diffractometer equipped with Pixcel detector using Nifiltered $\mathrm{Cu}-\mathrm{K} \alpha$ radiation $(0.154 \mathrm{~nm})$ in the range of $2 \theta=1-60^{\circ}$ and at a scan rate of 0.1 $\% / \mathrm{S}$. Morphological analyses of the parent zeolite and resulting ZTC samples were conducted using an Auriga Cobra Focused-Ion Beam Scanning Electron Microscope (FIB-SEM). The thermogravimetric analysis (TGA) curves were measured on a Mettler, Toledo, TGA/SDTA $851^{\mathrm{e}}$ instrument, the samples were heated to $\sim 1000{ }^{\circ} \mathrm{C}$ with heating rate of $10{ }^{\circ} \mathrm{C} / \mathrm{min}$ under air $(60 \mathrm{~mL} / \mathrm{min})$ and $\mathrm{N}_{2}$ flow was maintained at $40 \mathrm{~mL} / \mathrm{min}$ as a balance gas. $\mathrm{N}_{2}$ adsorption-desorption isotherms, pore size distributions (PSDs) and low-pressure $\mathrm{H}_{2}$ isotherms (up to 1 bar) at the temperature of liquid $\mathrm{N}_{2}$, were measured using a Micrometrics ASAP 2020 HD analyser. Before any gas adsorption experiment, the samples were outgassed in the degassing port under vacuum (down to $10^{-7}$ bar) for at least $8 \mathrm{~h}$ at $200{ }^{\circ} \mathrm{C}$ in order to remove any humidity and/or other physisorbed gases.

\section{Results and discussion}

The analysis of the carbonaceous gas fraction obtained from bubbling argon though the pyrolysis oil revealed that it contained some of the carbonaceous fraction originally found in the pyrolysis oil such as benzene, toluene, ethylbenzene, $\mathrm{m} / \mathrm{p}-\mathrm{Xylene}$, o-Xylene. This carbon rich fraction was utilised as the precursor for the synthesis of the templated carbons. The details of the results for analysis of pyrolysis oil and its vapour phase are available in the supplementary materials section (Figure S1, Tables S1 and S2). As for the structural and morphological analysis of the parent zeolite template and resulting ZTC samples, Figure 2 (a- 

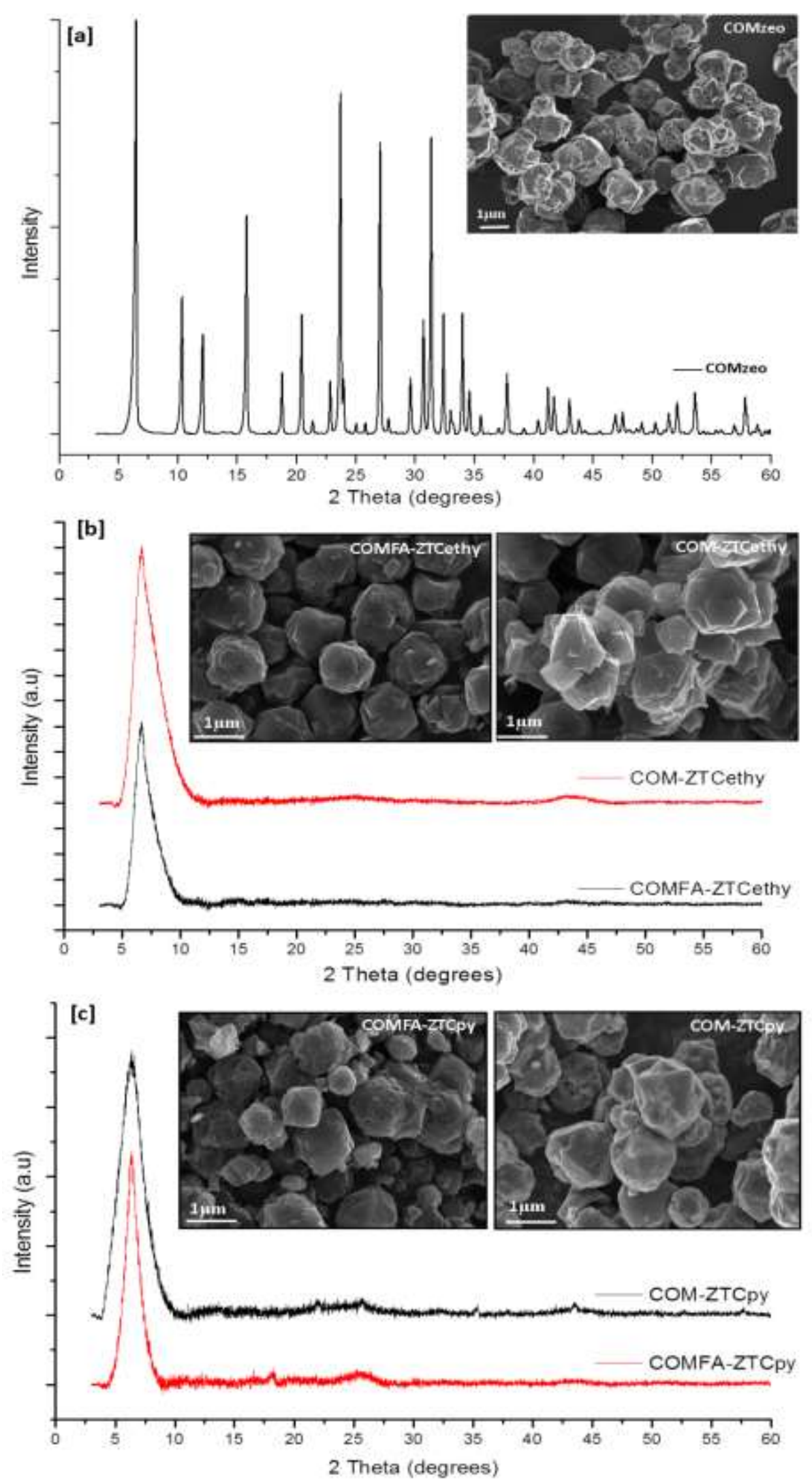

Figure 2: XRD patterns and SEM images (inset) of a) Zeolite X (COMzeo), b) ZTC obtained from use of ethylene gas from 1-step (COM-ZTCethy) and 2-step (COMFA-ZTCethy) c) ZTC obtained from use of pyrolysis vapour from 1-step (COM-ZTCpy) and 2-step (COMFA-ZTCpy). 
c) presents the XRD patterns together with their respective SEM images. The XRD patterns of all ZTC samples synthesized following either the 1 or 2-step process (Figure $2 \mathrm{~b}$ and c) exhibited the dominant peaks at about $2 \theta=6.3^{\circ}$, which is also present in the parent zeolite $13 \mathrm{X}$ (Figure 2 a). The appearance of this peak signifies resemblance of structural ordering similar to that of the parent zeolitic material and thus indicating success in the templating process. ${ }^{[10-}$ 13] The presence of a weak broad peaks at around $2 \theta=43^{\circ}$, especially for the 1-step ZTC sample obtained using ethylene gas, indicates that the sample had some graphitic components. This observation is thought to have occurred due to deposition of carbon on the near surface of the pores due spatial limitation on the porous structure of the zeolite. This deposition often leads to graphitization of the carbon during the carbonization step. ${ }^{[10,13,15]}$ In the respective SEM images of the synthesized ZTCs, the templated carbons show the octahedral pyramidal morphology which is similar to that of the parent zeolite. Noteworthy, there was no major morphological differences when either ethylene or pyrolysis oil vapour were used as the gaseous carbon precursor. This morphological resemblance of the parent material to that of the resulting ZTC is consistent with those obtained by other researchers. ${ }^{[6 \text {, }}$ $8,10,13,16-17]$

In order to complement the XRD results and determine the thermal stability of the obtained ZTCs, especially for those obtained using pyrolysis oil vapour, the TGA plots presented in Figure 3 show complete carbon burn off at around $600{ }^{\circ} \mathrm{C}$. This observation further indicates that the detemplation process was successful and the obtained ZTCs did not have any inorganic traces from the parent zeolite. ${ }^{[18,19]}$ The TGA of ZTCs synthesised using ethylene gas were earlier reported by Musyoka et al. ${ }^{[13]}$ 


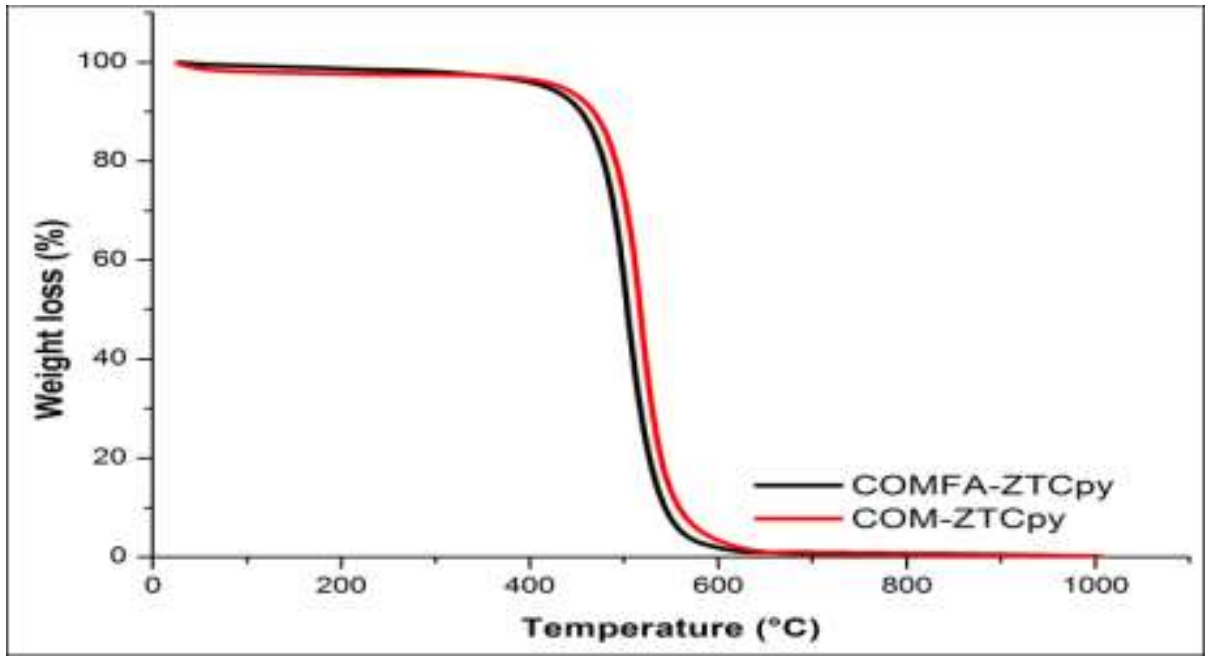

Figure 3: TGA plots for ZTCs synthesized using pyrolysis oil vapour.

Figure 4 presents the $\mathrm{N}_{2}$ sorption isotherms (at $77 \mathrm{~K}$ ) for both 1 and 2-step synthesis route for ethylene and pyrolysis oil vapour synthesised ZTCs. All the synthesised materials exhibited type IV isotherms (according to the IUPAC classification) with a steep $\mathrm{N}_{2}$ uptake at the lower partial pressures $\left(\mathrm{p} / \mathrm{p}_{0}<0.01\right)$ which suggests micropore filling. This observation is also consistent with previous reports that suggest that the micropore filling is an indication of successful replication of the zeolite structural ordering. ${ }^{[15,19]}$ The hysteresis loop observed at about $\mathrm{p} / \mathrm{p}_{0}>0.2$ can be associated with capillary condensation and signifies the presence of some mesoporosity. The presence of mesopores can either be associated with the presence of interparticle voids or occur as a result of partial carbon infiltration in the nano-channels of the zeolite. ${ }^{[13,17,20]}$ The textural properties of the synthesized ZTCs samples are listed in Table 1. The 2-step synthesized carbons had the highest specific surface area. In this case, COMFAZTCethy had the surface area of $3341 \mathrm{~m}^{2} / \mathrm{g}$ and also possessed the largest micropore volume of $1.72 \mathrm{~cm}^{3} / \mathrm{g}$ whereas COMFA-ZTCpy had a specific surface area of $3254 \mathrm{~m}^{2} / \mathrm{g}$ with micropore volume of $1.61 \mathrm{~cm}^{3} / \mathrm{g}$. These results show that in the 2-step method, the structural ordering is dependent on the carbon precursor that is introduced during the CVD step. This phenomenon was explained by Xia et al where they had reported ZTCs synthesised by 
impregnation with FA and different gas precursors. ${ }^{[21]}$ In the 1-step synthesis, COMZTCethy (ethylene synthesised) had a specific surface area of $2765 \mathrm{~m}^{2} / \mathrm{g}$ with micropore volume of $1.41 \mathrm{~cm}^{3} / \mathrm{g}$ whereas COM-ZTCpy (pyrolysis oil vapour synthesised) had specific surface area of $2189 \mathrm{~m}^{2} / \mathrm{g}$ with micropore volume of $1.25 \mathrm{~cm}^{3} / \mathrm{g}$. The overall specific surface area results are consistent with previous reports that had reported that surface areas of ZTCs are often within the range reported in this study. ${ }^{[11,14,18,22]}$

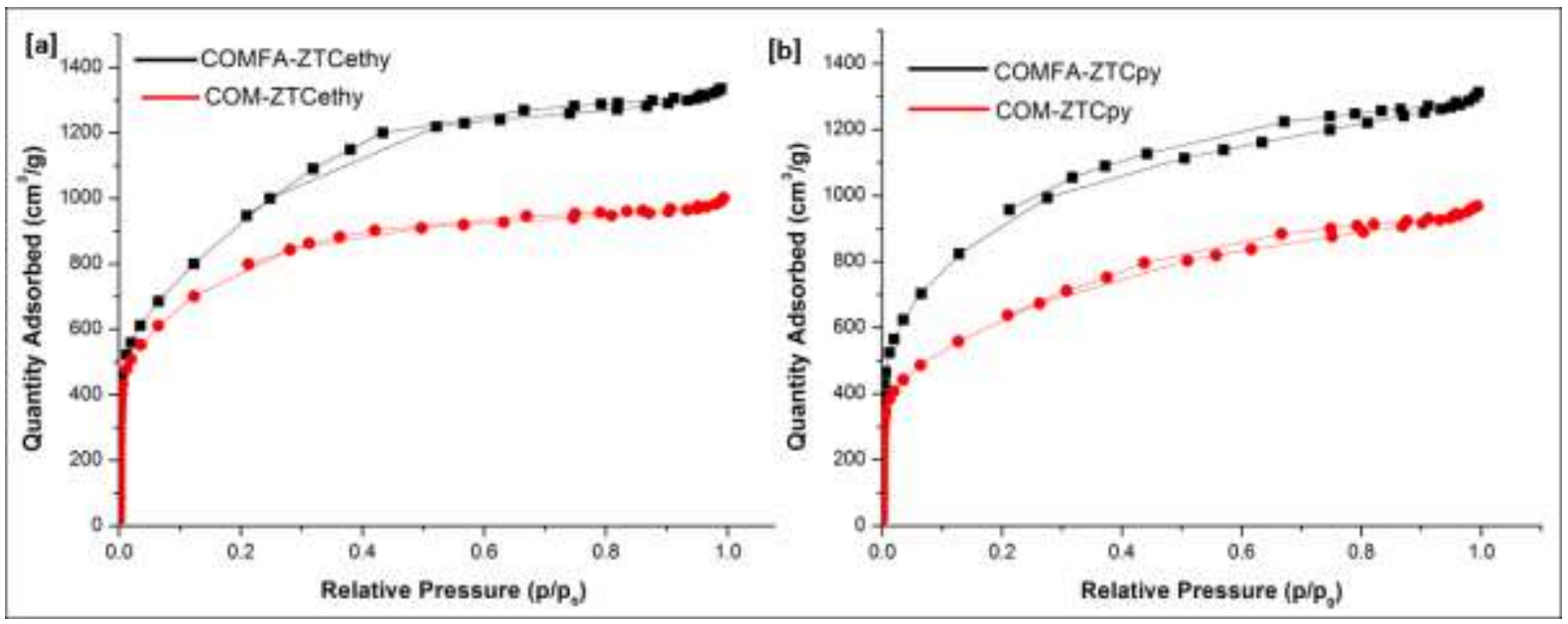

Figure 4: N2 sorption isotherms of ZTCs synthesized using a) ethylene gas and b) pyrolysis oil vapour.

Table 1: Textural properties of ZTC obtained from the use of ethylene gas and pyrolysis oil vapour.

\begin{tabular}{|c|c|c|c|c|}
\hline Sample Name & $\begin{array}{c}\text { BET Specific surface } \\
\text { area }\left(\mathrm{m}^{2} / \mathrm{g}\right)\end{array}$ & $\begin{array}{c}\text { Micropore Volume } \\
\left(\mathrm{cm}^{3} / \mathrm{g}\right)\end{array}$ & $\begin{array}{c}\text { Pore size } \\
(\mathrm{nm})\end{array}$ & $\begin{array}{c}\text { Pore Volume } \\
\left(\mathrm{cm}^{3} / \mathrm{g}\right)\end{array}$ \\
\hline COMFA-ZTCethy & 3341 & 1.72 & 1.28 & 1.88 \\
\hline COMFA-ZTCpy & 3254 & 1.61 & 1.23 & 1.40 \\
\hline COM-ZTCethy & 2765 & 1.41 & 1.22 & 1.24 \\
\hline COM-ZTCpy & 2189 & 1.25 & 1.21 & \\
\hline
\end{tabular}


From Table 1, it can be noted that ZTCs porous properties (specific surface areas, micropore volumes and pore diameters) are dependent on the synthesis conditions. These attributes were also reported to be dependent on the structural regularity and degree of graphitization of the obtained carbons. ${ }^{[14]}$ Overall, the ZTCs synthesized using ethylene had larger specific surface area as compared with the pyrolysis oil vapour synthesized ZTCs. This suggests that the type of carbon precursor used has an important role in the structural regularity of the resultant carbons. This could be due to the fact that ethylene consists of a rich carbon content of similar chemical composition unlike pyrolysis oil that consists of different molecules with different concentrations. The results also shows that the FA impregnation assists in boosting carbon loading and structural regularity which then generates more ordered porosity when a rich carbon precursor is used. ${ }^{[22]}$

Figure. 5 presents the pore size distribution plots generated according to the HorvathKawazoe (H-K) method. All the ZTCs exhibited a pore size distribution that was dominant at around $1.2 \mathrm{~nm}$. The pore sizes are consistent with previous reported results that indicated that ZTCs with structural ordering of zeolites do not possess pores with sizes larger than $1.5 \mathrm{~nm}$. ${ }^{[15]}$ The ZTCs obtained using pyrolysis oil vapours were noted to have a narrower pore size distribution. Pore splitting, at the pore range of $1.2-1.27 \mathrm{~nm}$, was observed for 1-step synthesised materials (COM-ZTCethy and COM-ZTCpy) which confirmed presence of both smaller and larger pores at the micropore region. The 2-step synthesized samples did not show this pore splitting characteristic and this observation may be due to the blockage of pores during FA impregnation and polymerization process. 


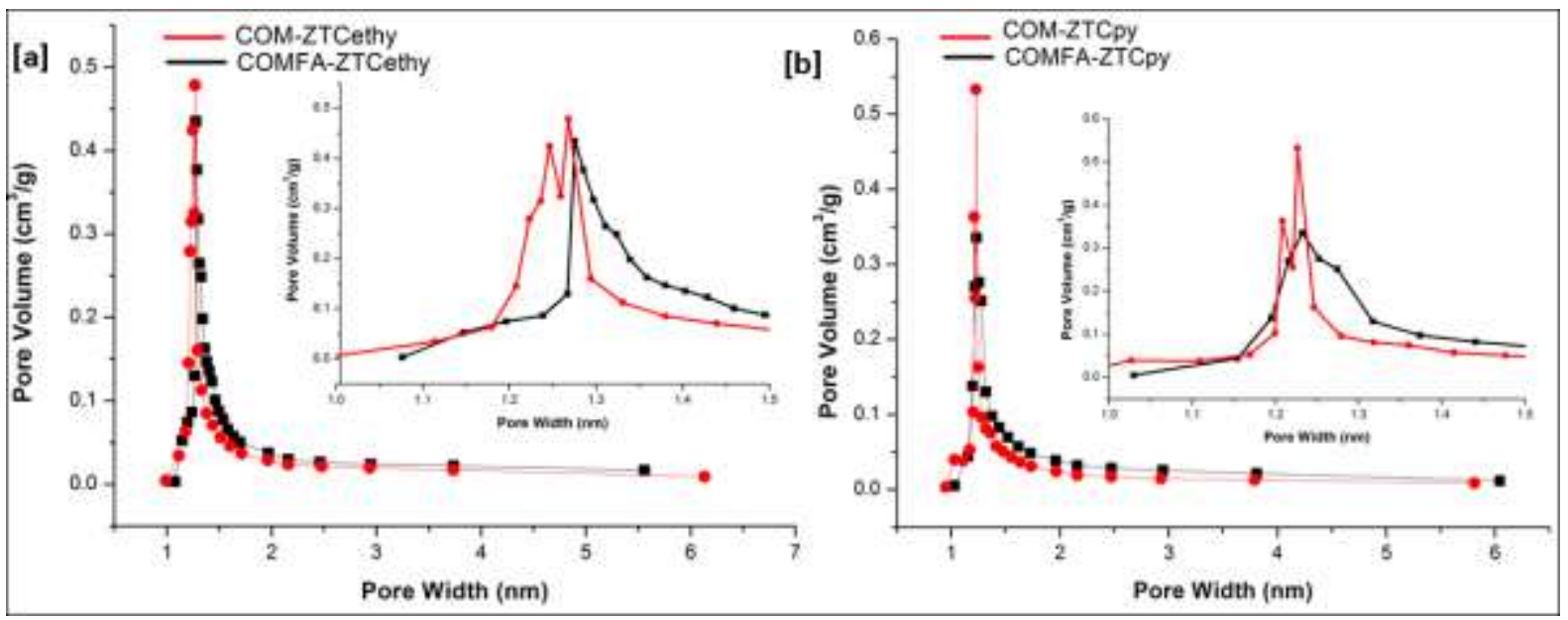

Figure 5: PSD for ZTCs synthesised using a) ethylene gas and b) pyrolysis oil vapour.

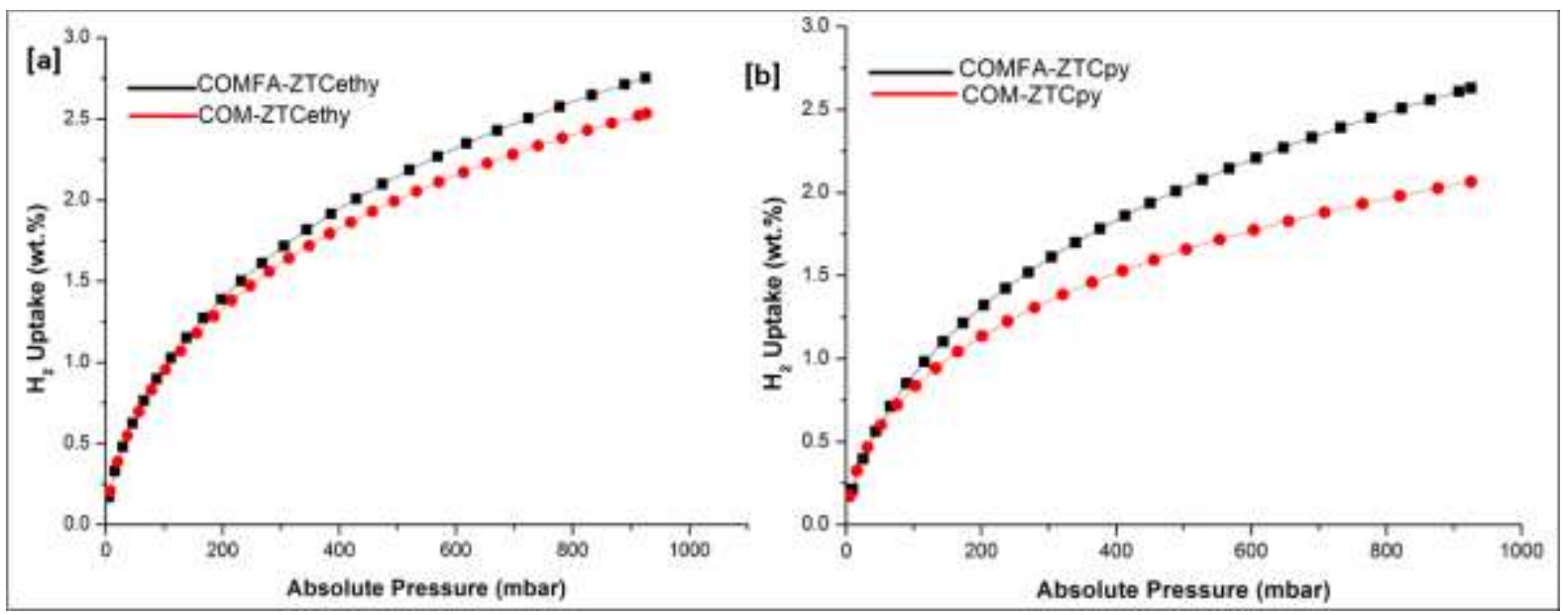

Figure 6: H2 uptake for ZTCs synthesised using a) ethylene gas and b) pyrolysis oil vapour

The $\mathrm{H}_{2}$ sorption isotherms are presented in Figure 6. The isotherms confirm that hydrogen uptake was completely reversible since there was no hysteresis loop. There is also no observable saturation at 1 bar which suggests that obtained ZTC materials can adsorb more hydrogen at relatively higher pressures. The ability of a material to adsorb hydrogen is associated with the specific surface area, the micropores and the pore volume. ${ }^{[6]}$ COMFAZTCethy sample adsorbed more $\mathrm{H}_{2}$ at 1 bar because it had the highest specific surface area and pore volume. As the textural properties decreases, there is also a decrease in the 
adsorption of $\mathrm{H}_{2}$ from 2.5 to 1.9 wt. $\%$. These results are consistent with previous reports. ${ }^{[6,}$ 13-15, 18, 22]

Since the results presented in this study are those obtained from the use of gas bubbled through the pyrolysis oil, studies on the use of directly tapped pyrolysis gas (pyro-gas) which is obtained during the waste tyre pyrolysis are recommended for the near future. Due to the fact that the non-condensable pyro-gases are essentially composed of hydrogen, carbon monoxide, carbon dioxide, methane, ethane, butadiene and other light carbonaceous molecules, their use in the production of nanocasted/templated carbons can be expected to be successful. Experimental work will further confirm this hypothesis.

\section{Conclusion}

In conclusion, the use of the waste tyres pyrolysis oil as a carbon precursor in the synthesis of ZTCs proved to be successful. The synthesized ZTC had high specific surface areas that were comparable to those synthesized using commercial ethylene gas. Importantly, high hydrogen uptake capacities were also obtained for the ZTC samples and were also comparable to other prior studies that had utilised commercially sourced carbon rich gases. Future studies will investigate the use of directly tapped pyrolysis gas (pyro-gas) obtained during the waste tyre pyrolysis process to produce ZTCs and/or other types of templated carbons. 


\section{Acknowledgement}

The authors of this paper acknowledge and thank the South African Department of Science and Technology (DST) for the financial support towards HySA Infrastructure (Grant No. HTC004X), the Council for Scientific and Industrial Research (CSIR) for providing facilities and National Research Foundation (NRF) for funding the SA-Poland collaborative project (HTC071X). Any opinions, findings and/or recommendations expressed here are those of the authors and not of the funding bodies.

\section{References}

[1] Williams, P.T. Pyrolysis of waste tyres: a review. Waste Manage. 2013, 33 (8), 17141728.

[2] Ko, D.C.; Mui, E.L.; Lau, K.S.; McKay, G. Production of activated carbons from waste tire-process design and economical analysis. Waste Manage. 2004, 24 (9), 875-888.

[3] Juma, M.; Koreňová, Z.; Markoš, J.; Annus, J.; Jelemenský, L. Pyrolysis and combustion of scrap tire. Pet. Coal. 2006, 48 (1), 15-26.

[4] Martínez, J.D.; Murillo, R.; García, T.; Arauzo, I. Thermodynamic analysis for syngas production from volatiles released in waste tire pyrolysis. Energy Convers. Manage. 2014, $81,338-353$.

[5] Stadie, N.P.; Wang, S.; Kravchyk, K.V.; Kovalenko, M.V. Zeolite-templated carbon as an ordered microporous electrode for aluminum batteries. ACS nano. 2017, 11 (2), 1911-1919. 
[6] Yang, Z.; Xia, Y.; Mokaya, R. Enhanced hydrogen storage capacity of high specific surface area zeolite-like carbon materials, J. Amer. Chem. Soc. 2007, 129 (6), 1673-1679.

[7] Xie, Y.; Kocaefe, D.; Chen, C.; Kocaefe, Y. Review of Research on Template Methods in Preparation of Nanomaterials. J. Nanomater Article. 2016,1-10. ID 2302595.

[8] Ma, Z.; Kyotani, T.; Tomita, A. Synthesis methods for preparing microporous carbons with a structural regularity of zeolite Y. Carbon. 2002, 40 (13), 2367-2374.

[9] Yang, Z.; Xiong, W.; Wang, J.; Zhu, Y.; Xia, Y. A Systematic Study on the Preparation and Hydrogen Storage of Zeolite 13X-Templated Microporous Carbons. Eur. J. Inorg. Chem. 2016, (13-14), 2152-2158.

[10] Xia, Y.; Mokaya, R.; Grant, D.M.; Walker, G.S. A simplified synthesis of N-doped zeolite-templated carbons, the control of the level of zeolite-like ordering and its effect on hydrogen storage properties. Carbon. 2011, 49 (3), 844-853.

[11] Kyotani, T.; Ma, Z.; Tomita, A. Template synthesis of novel porous carbons using various types of zeolites. Carbon. 2003, 41 (7), 1451-1459.

[12] Xia, Y.; Yang, Z.; Mokaya, R. CVD Nanocasting Routes to Zeolite-Templated Carbons for Hydrogen Storage. Chem. Vap. Deposition. 2010, 16 (10-12), 322-328. 
[13] Musyoka, N.M.; Ren, J.; Langmi, H.W.; North, B.C.; Mathe, M. A comparison of hydrogen storage capacity of commercial and fly ash-derived zeolite $\mathrm{X}$ together with their respective templated carbon derivatives. Int. J. Hydrogen Energy. 2015, 40 (37), 1270512712.

[14] Nishihara, H.; Hou, P.X.; Li, L.X.; Ito, M.; Uchiyama, M.; Kaburagi, T.; Kyotani, T. High-pressure hydrogen storage in zeolite-templated carbon. J. Phys. Chem. C. 2009, 113 (8), 3189-3196.

[15] Masika, E.; Mokaya, R. Preparation of ultrahigh specific surface area porous carbons templated using zeolite $13 \mathrm{X}$ for enhanced hydrogen storage. Prog. Nat. Sci. Mater.Int. 2013, $23(3), 308-316$.

[16] Kyotani, T.; Nagai, T.; Inoue, S.; Tomita, A. Formation of new type of porous carbon by carbonization in zeolite nanochannels. Chem. Mater. 1997, 9 (2), 609-615.

[17] Song, X. H.; Xu, R.; Wang, K. The structural development of zeolite-templated carbon under pyrolysis J. Anal. Appl. 2013, 100, 153-157.

[18] Alam, N.; Mokaya, R. Evolution of optimal porosity for improved hydrogen storage in templated zeolite-like carbons. Energy Environ. Sci. 2010, 3 (11), 1773-1781.

[19] Pacuła, A.; Mokaya, R. Synthesis and high hydrogen storage capacity of zeolite-like carbons nanocast using as-synthesized zeolite templates. J. Phys. Chem. C. 2008, 112 (7), 2764-2769. 
[20] Guan, C.; Wang, K.; Yang, C.; Zhao, X.S. Characterization of a zeolite-templated carbon for $\mathrm{H}_{2}$ storage application. Microporous Mesoporous Mater. 2009, 118 (1-3), 503-507.

[21] Xia, Y.; Yang, Z.; Mokaya, R. CVD Nanocasting Routes to Zeolite-Templated Carbons for Hydrogen Storage. Chem. Vap. Deposition. 2010, 16 (10-12), 322-328.

[22] Yang, Z.; Xiong, W.; Wang, J.; Zhu, Y.; Xia, Y. A Systematic Study on the Preparation and Hydrogen Storage of Zeolite 13X-Templated Microporous Carbons, Eur. J. Inorg. Chem. 2016, (13-14), 1434-1948.

\section{Supporting Information}

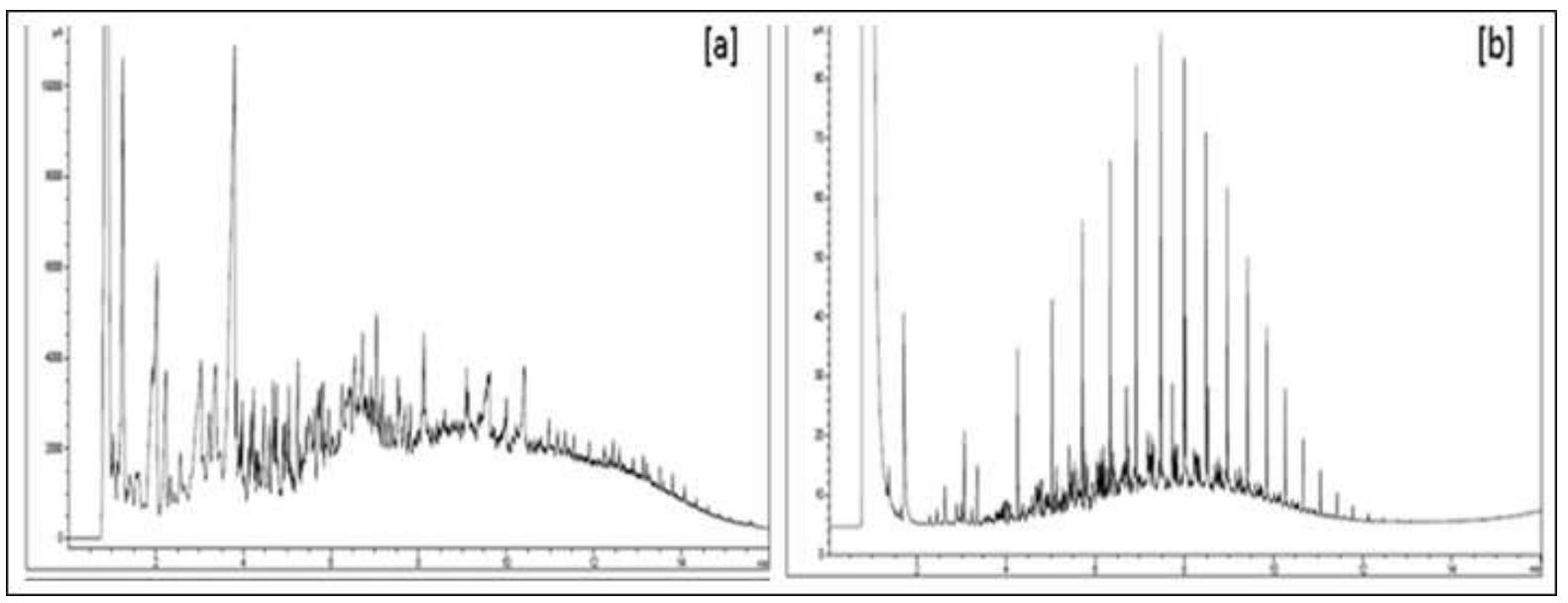

Figure S1: Comparison of GC profile spectra of a) pyrolysis oil b) Commercial diesel. 
Table S1: Pyrolysis oil constituents.

\begin{tabular}{|c|c|}
\hline Composition & Percentage composition (\%) \\
\hline Tolune & 8 \\
\hline Ethylbenzene & 5 \\
\hline $\mathrm{m} / \mathrm{p}$-xylene & 8 \\
\hline Isopropylbenzene & 1 \\
\hline Propylbenzene & 4 \\
\hline D-Limonene & 12 \\
\hline 1,2,3,4-Tetramethylbenzene & 1 \\
\hline Benzothiazole & 2 \\
\hline Naphthalene,1,7-dinethyl & 2 \\
\hline 2,4-Dimethylquinoline & 2 \\
\hline 1,6,7-Trimethylnaphthalene & 1 \\
\hline n-Hexadecanoic acid & 2 \\
\hline N-1,3-Dimethylbutyl-N'-phenyl-1,4-benzenediamine & 5 \\
\hline
\end{tabular}

Table S2: BTEX analysis results.

\begin{tabular}{|l|l|l|l|l|l|}
\hline Sample ID & $\begin{array}{l}\text { Benzene } \\
(\mathbf{n g} / \boldsymbol{\mu L})\end{array}$ & $\begin{array}{l}\text { Toluene } \\
(\mathbf{n g} / \boldsymbol{\mu L})\end{array}$ & $\begin{array}{l}\text { Ethylbenzene } \\
(\mathbf{n g} / \boldsymbol{\mu L})\end{array}$ & $\begin{array}{l}\mathbf{m} / \mathbf{p}-X y l e n e \\
(\mathbf{n g} / \boldsymbol{\mu L})\end{array}$ & $\begin{array}{l}\text { o-Xylene } \\
(\mathbf{n g} / \boldsymbol{\mu L})\end{array}$ \\
\hline PYG1 O-16-01755 & 570 & 1200 & 260 & 230 & 120 \\
\hline PYG2 O-16-01756 & 510 & 1100 & 240 & 210 & 110 \\
\hline PYG3 O-16-01757 & 660 & 1200 & 300 & 290 & 150 \\
\hline Average & 580 & 1167 & 267 & 243 & 127 \\
\hline
\end{tabular}

